Methods: An electronic diary (ED) run on mobile phones was trailed with eight young people recruited from a youth health clinic that regularly drank alcohol. The ED assessed alcohol use, mood, stresses and activities. Participants were asked to complete the ED four times each day for 1 week and provide feedback about the diary's effectiveness and ease of use.

Results: Drinking alcohol was associated with cooking/eating and study/homework. While drinking, participants were mostly alone $(33.3 \%)$ or with their partner $(33.3 \%)$ and drank to relax $(33.3 \%)$ or feel good (16.7\%). Participants generally had low negative mood while drinking and drank from 0 to 5 times a week.

Conclusions: After some setbacks, the ED was successfully implemented in a clinical setting. Responses to the diary provided a rich variety of information including information on mood, stresses, activities, alcohol and marijuana use. Participants' feedback on how they found the diary was also useful and provided good suggestions on how to improve the diary for further studies.

\section{The SHADE Project: self-help for alcohol/other drug use and depression}

\author{
F Kay-Lambkin', V Carrr, ${ }^{1,2}$, Lewin' ${ }^{1}$, B Kelly ${ }^{3}$, A Baker ${ }^{1}$ \\ ${ }^{1}$ Centre for Mental Health Studies, University of Newcastle; ${ }^{2}$ NISAD; and \\ ${ }^{3}$ Centre for Rural and Remote Mental Health, New South Wales, Australia
}

Background: The co-occurrence of depression and alcohol/other drug misuse is more common than expected by chance alone. Despite this, an effective program of treatment is yet to be established for people experiencing this comorbidity. This is a concern, given rates of depression and alcohol misuse are on the increase.

Aim: This paper will report on the posttreatment alcohol/other drug- and depression-related outcomes of the SHADE project, a large-scale, multisite study of computerized psychological treatment.

Methods: SHADE participants were those with current levels of depression and current problematic use of alcohol, cannabis or amphetamines. Following an initial assessment, participants received one face-toface case formulation session with a therapist and were subsequently randomized to receive nine sessions of SHADE therapy through a therapist, nine sessions of SHADE therapy through a computer or nine sessions of person-centered (supportive) counseling. Follow-up occurred at posttreatment, 6- and 12-month follow-up.

Results: Posttreatment results will be reported for the 250 participants recruited to the study in rural/remote and urban NSW.
Conclusions: Computerized treatment is not meant as a stand-alone therapy. The results from this study suggest that computer-based interventions can produce important gains for people with depression and alcohol/other drug use comorbidity. Further implications will be discussed.

\section{Mental health first aid standards for self-harm}

\author{
C Kelly, R Langlands, A Jorm
}

ORYGEN Research Centre, Melbourne, Australia

Background: Mental disorders are highly prevalent, but many people do not seek help for them. We aim to develop mental health first aid standards for a number of mental disorders and related crises. Deliberate, nonsuicidal self-injury is becoming more common, particularly among young people. Little is known about how to prevent repetition or support someone who is deliberately harming him/herself.

Methods: The methodology of this study is the 'Delphi method', a technique for determining consensus. Initially, we searched for claims and suggestions about how to help someone who engages in deliberate selfharm. Searching the medical literature, carer's manuals, memoirs, pamphlets and Web sites, very little was found. Round 1 of this study instead asked people to write in their own words what might be the right thing to do. Questions have been generated from these written answers. Respondents are clinicians or researchers, carers and consumers from Australia, New Zealand, Canada, the UK and the United States.

Results: The majority of the participants felt that 'stopping someone' from injuring him/herself is counterproductive. The two emphases are on dealing medically with any serious injuries and allowing the person space to talk about his/her distressing feelings.

Conclusions: These standards will be used in Mental Health First Aid training and will be widely available to members of the public who are concerned about a family member or friend who is engaging in self-injury.

\section{Vocational rehabilitation in first- episode psychosis: results of the first Australian randomized controlled trial of individual placement and support}

\section{E Killackey 1,2, P McGorry ${ }^{1,3}$, K Roffel', G Chinnery', H Jackson ${ }^{2}$}

'ORYGEN Research Centre; 2Department of Psychology, The University of Melbourne; and ${ }^{3}$ ORYGEN Youth Health, Melbourne, Australia 